\title{
Tibial Tubercle-Midepicondyle Distance Can Be a Better Index to Predict the Outcome of Medial Patellofemoral Ligament Reconstruction Than Tibial Tubercle-Trochlear Groove Distance
}

\author{
Tomoya Iseki, M.D., Hiroshi Nakayama, M.D., Takashi Daimon, Ph.D., \\ Shunichiro Kambara, M.D., Ryo Kanto, M.D., Motoi Yamaguchi, M.D., \\ Shintaro Onishi, M.D., Toshiya Tachibana, M.D., and Shinichi Yoshiya, M.D.
}

\begin{abstract}
Purpose: To compare the clinical utility of tibial tubercle-midepicondyle (TT-ME) and tibial tubercle-trochlear groove (TT-TG) distances in predicting the risk for recurrent instability after isolated MPFL reconstruction. Methods: A consecutive series of patients with recurrent patellar dislocation who underwent isolated MPFL reconstruction made up the study population. The patients were followed for a minimum of 2 years. In assessment of surgical outcome, the patient was deemed to exhibit "postoperative recurrent patellar instability" when $\geq 1$ of the following 3 conditions was identified: redislocation, positive apprehension sign, and positive J-sign (manifestation of abnormal patellar tracking). As for radiological parameters for position of the tibial tubercle, TT-ME distance (transverse distance between the tibial tubercle and midpoint of the transepicondylar line) and TT-TG distance were measured on axial computed tomography images. The clinical utility as a factor to predict the outcome of MPFL reconstruction was compared between the 2 distances using receiver operating characteristic (ROC) analysis. In addition, various radiological indices potentially influencing the surgical outcome were subjected to multivariable logistic regression analysis. Results: We examined 38 knees in 38 patients with a mean age at surgery of 17.6 years. Postoperative recurrent patellar instability was encountered in 8 of the 38 knees. The ROC curve analysis showed the TT-ME distance to be a significantly better indicator in predicting surgical outcome than the TT-TG distance $(P=.001)$. The univariate analysis for radiological factors demonstrated that the TT-ME distance was significantly associated with postoperative recurrent patellar instability (odds ratio $1.42, P=.012$ ) whereas all other factors including the TT-TG distance did not correlate with recurrent instability. The multivariable logistic regression analysis revealed that only the TT-ME distance was significantly associated with recurrent instability $(P=.035)$. Conclusions: Analysis of our patient population undergoing isolated MPFL reconstruction showed that the TT-ME distance was a significantly better indicator than the TT-TG distance to predict the risk for recurrent instability after isolated MPFL reconstruction performed for patellar instability. Level of Evidence: Level IV, therapeutic case series.
\end{abstract}

\section{Introduction}

$\mathbf{M}$ edial patellofemoral ligament (MPFL) reconstruction is widely adopted as a surgical option for knees with patellar instability, and satisfactory results have been reported in the majority of previous

From the Department of Orthopaedic Surgery, Hyogo College of Medicine, Nishinomiya, Japan (T.I., H.N., R.K., S.O., T.T.), the Department of Biostatistics, Hyogo College of Medicine, Nishinomiya, Japan (T.D.), the Department of Orthopaedic Surgery, Nishinomiya Kaisei Hospital, Nishinomiya, Japan (S.K.), and the Department of Orthopaedic surgery, Meiwa Hospital, Nishinomiya, Japan (M.Y., S.Y.)

The authors report that they have no conflicts of interest in the authorship and publication of this article. Full ICMJE author disclosure forms are available for this article online, as supplementary material.

Received June 26, 2019; accepted April 29, 2020. studies. ${ }^{1-5}$ However, restoration of soft tissue restraint with MPFL reconstruction alone is not enough to stabilize the patella in knees with combined bony pathologies, such as lateralized tibial tuberosity.,6-15 Conventionally, the tibial tubercle-trochlear groove

Address correspondence to Shinichi Yoshiya, M.D., Nishinomiya Kaisei Hospital, 1-4 Ohama-cho, Nishinomiya, Hyogo 662-0957, Japan. E-mail: yoshiya0307@gmail.com

(C) 2020 THE AUTHORS. Published by Elsevier Inc. on behalf of the Arthroscopy Association of North America. This is an open access article under the CC BY-NC-ND license (http://creativecommons.org/licenses/by-nc-nd/4.0/). 2666-061X/19755

https://doi.org/10.1016/j.asmr.2020.04.014 
(TT-TG) distance has been used as an index for assessing the position of the tibial tubercle, ${ }^{10-12}$ and increased TT-TG distance has been regarded as a factor impairing the outcome of isolated MPFL reconstruction. Therefore, for knees with a large TT-TG value, tibial tubercle transfer was adopted as a surgical option either alone or in combination with MPFL reconstruction. ${ }^{16-18}$

To date, however, there has been no consensus on an absolute cutoff value in determining surgical indication. ${ }^{13-15}$ In addition, as for measurement accuracy, reliability of the TT-TG distance assessment can be impaired in knees with dysplastic trochlea because of the difficulty of localizing the proximal reference point (deepest point of the trochlear groove). Moreover, medial deviation of the trochlear groove associated with hypoplastic lateral trochlea, which is often observed as another bony pathology, may result in overestimation of the TT-TG distance.

In this article, we propose a morphological index: tibial tubercle-midepicondyle (TT-ME) distance. The midpoint of the transepicondylar line can be used as a reference point for the femoral trochlea to create the TT-ME distance index. Using this proximal reference point, the potential problem regarding the reliability of the TT-TG measurement as mentioned above may be avoided, enabling more accurate evaluation of the lateralization of the tibial tubercle.

The purpose of this study was to compare the clinical utility of TT-ME and TT-TG distances in predicting the risk for recurrent instability after isolated MPFL reconstruction. It was hypothesized that the TT-ME distance can be a more reliable indicator to predict the outcome of isolated MPFL reconstruction for knees with patellar instability compared with the TT-TG distance.

\section{Methods}

\section{Study Population}

This study was designed as a retrospective analysis of a case series. A consecutive series of patients who underwent MPFL reconstruction between April 2011 and March 2015 were initially enrolled in the study, and the patient records were reviewed. Inclusion criteria were knees with recurrent patellar dislocation or continued symptomatic instability with a positive apprehension sign. Exclusion criteria were combined MPFL reconstruction and tibial tubercle transfer, as well as knees with open physis for whom surgical procedures were different from that described in this article. During the study period, MPFL reconstruction was our principle surgical option. Surgery was indicated after failed conservative treatments with muscle exercise and bracing. In knees with the TT-TG distance $>22 \mathrm{~mm}$, combined MPFL reconstruction and tibial tubercle transfer was adopted as the performed procedure.
The study was approved by the institutional review board of our institution (registration number 2221), and informed consent was obtained from each patient included in the study.

\section{Surgical Technique}

MPFL reconstruction was performed using autogenous semitendinosus tendon harvested from the ipsilateral leg. The harvested tendon was made into a double-stranded graft for reconstruction. For preparation of the graft fixation sites, longitudinal skin incisions were made 3 to $5 \mathrm{~cm}$ in length at the medial margin of the patella and the medial epicondyle of the femur. Two sockets for anchor insertion were prepared at the proximal and center portions of the medial aspect of the patella using a 2.9-mm-diameter pin attached to the anchor system. Subsequently, 2 suture anchors (Super QuickAnchor Plus DS; DePuy Mitek, Raynham, MA) for graft fixation on the patellar side were inserted into the sockets. A guide wire was inserted at the tentative femoral fixation site between the medial epicondyle and the adductor tubercle, and No. 2 nonabsorbable high-strength sutures attached to the anchors were passed between the second and third layers of the medial supporting structure and looped over the guide wire. To examine the change in graft length through the range of motion, the knee was moved from maximum extension to deep flexion while traction was manually applied to the suture. When the examined length change pattern was not acceptable, the position of the guide wire was changed until the optimal femoral attachment site exhibiting slight elongation in extension could be identified. Once the appropriate attachment site was located, the guide wire was overreamed with a 6-mm cannulated reamer to the depth of $25 \mathrm{~mm}$.

Graft fixation on the femoral side was achieved with a 6-mm interference screw. Thereafter, the other end of the tendon graft was fixed to the patella using suture anchors inserted as described above. The No. 2 highstrength suture attached to the anchor was sewed into each of the 2 free ends of the double-stranded graft using Krackow's stitch technique. During fixation, manual tension was applied to the tendon graft while the patella was centered with the knee in $20^{\circ}$ to $30^{\circ}$ of flexion. The periosteum of the patella and the surrounding soft tissue were further sutured over the end of the tendon graft to supplement the fixation. Surgeries were performed by either of 2 experienced surgeons (M.Y. and H.N.).

\section{Postoperative Rehabilitation}

Knees were immobilized in extension for 1 week after surgery. Range of motion exercise was started at 1 week. Partial weightbearing was allowed at 1 week, with progression to full weightbearing at 3 to 4 weeks. 
For patients who could not stably bear the weight on the operated leg, use of crutches or a knee immobilizer during walking was instructed, and permission for full weightbearing was delayed. Jogging was begun at 3 months, and full return to strenuous sports activity was permitted between 6 and 9 months.

\section{Radiological Evaluation}

Routine radiological and computed tomography (CT) examinations were performed for all knees preoperatively with the consent of each patient and the patient's family. A 64-slice multidetector CT scanner (SOMATOM Definition Edge; Siemens, Munich, Germany) was used. The slice thickness was set at $0.7 \mathrm{~mm}$ for all examined knees. Knees were placed in full extension during image acquisition.

Evaluation of the TT-TG distance followed the measurement method proposed and used in a previous study. ${ }^{11}$ The axial CT images at the levels including the deepest point of the trochlear groove and the highest point of the tibial tuberosity were superimposed using OsiriX MD software (Pixmeo, Geneva, Switzerland), and the transverse distance between the bottom of the femoral groove and the highest point of the tibial tubercle was measured using the posterior condylar line as a reference (Fig $1 \mathrm{~A}-\mathrm{C}$ ). The TT-ME distance was defined as the transverse distance between the highest point of the tibial tubercle and the midpoint of the transepicondylar line. The transepicondylar line was determined on the axial image by connecting the most prominent points of the medial/lateral epicondyles. As in the TT-TG measurement, the axial images at the levels of the most prominent points of the medial/ lateral epicondyles and the tibial tuberosity were superimposed, and the transverse distance between the 2 points was measured using the transepicondylar line as a reference (Fig 1D-F).

In addition, other conventional radiological indices, such as the sulcus angle, congruence angle, tilting angle/lateral shift of the patella, Insall-Salvati ratio, and Caton-Deschamps ratio, were measured on radiographs and CT images. Trochlear dysplasia was classified into 4 types using the Dejour classification. ${ }^{1,14}$ Image analysis was conducted by one of the authors (T.I.), who was
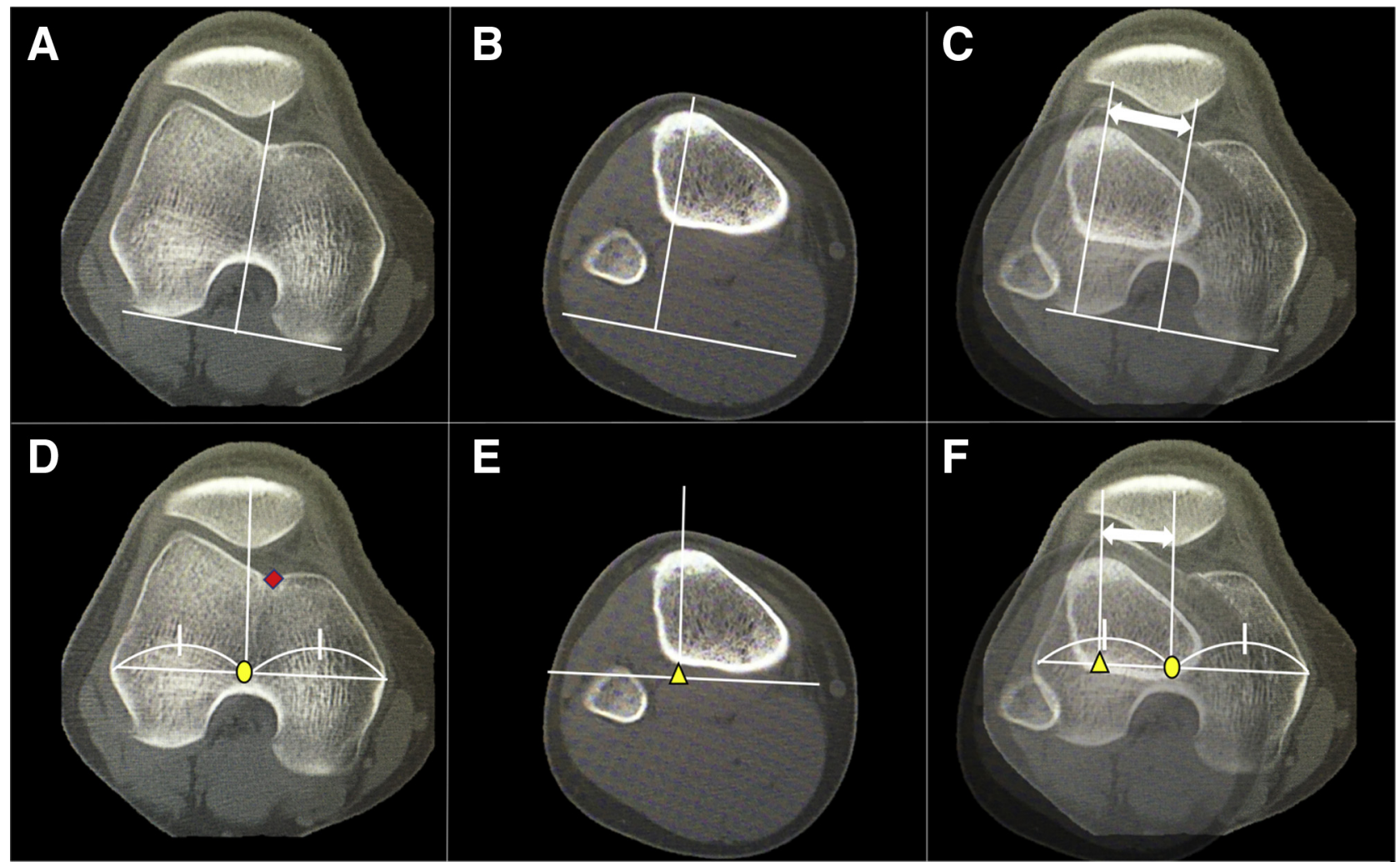

Fig 1. Computed tomography measurement methods for lateralization of the tibial tubercle. Tibial tubercle-trochlear groove (TTTG) distance: The measurement is made as described in the literature. ${ }^{11}$ The transverse distance between the bottom of the femoral groove (A) and the highest point of the tibial tubercle (B) was measured using the posterior condylar line as a reference (C). Tibial tubercle-midepicondyle (TT-ME) distance: (D) The midpoint of the transepicondylar line (yellow dot) is identified as a proximal reference. This line is not affected by deepest point of trochlear femoral groove (red rhombus). (E) A vertical line to the tibial tubercle from the transepicondylar line is drawn (yellow arrowhead). (F) TT-ME distance represents the transverse distance between the midpoint of the transepicondylar line (yellow dot) and the tibial tubercle (yellow arrowhead) on the superimposed axial computed tomography image. 
not involved in surgery or clinical assessment of the study population.

\section{Postoperative Clinical Evaluation and Definition of Recurrent Patellar Instability}

After surgery, patients were periodically followed up every 3 months within a year and subsequently at 18 and 24 months, followed by yearly checkups. Satisfactory attainment of patellar stability was defined by the following 3 conditions: (1) no recurrent dislocation, (2) negative apprehension sign, and (3) negative J-sign (visual identification of excessive lateral shift of the patella during terminal active knee extension). If $\geq 1$ of these 3 conditions were not attained, the operated knee was deemed as having recurrent patellar instability, indicating failure to completely restore patellar stability and tracking.

\section{Statistical Analysis}

Continuous variables are summarized as means with standard deviation or range (minimum to maximum). Categorical variables are summarized as frequencies with percentages. For comparison between TT-ME and TT-TG distances for ability in differentiating knees with satisfactory patellar stability and recurrent instability, receiver operating characteristic (ROC) curves were used. The optimal cutoff value was identified, and the area under the ROC curve (AUC) was compared between the 2 distances. In assessment of the association of postoperative recurrent patellar instability with radiological (potential prognostic) factors, a univariable analysis was initially performed. Factors that were found to have values of $P<.1$ in the univariable analysis were further analyzed in the multivariable logistic regression analysis. Results are summarized as odds ratios with their corresponding 95\% confidence intervals and $P$ values. All $P$ values were 2 -sided, and $P$ $<.05$ was considered statistically significant. The statistical analyses were performed using SPSS (version 19, SPSS, Chicago, IL) and R (version 3.2.0).

\section{Results}

\section{Patient Population and Profiles}

During the study period, in total, 50 knees in 48 patients underwent MPFL reconstruction. Among those initially enrolled subjects, combined MPFL reconstruction and tibial tubercle transfer was performed on 2 knees, and 2 knees had apparent open physis. Those 4 knees were excluded from the study, leaving 46 knees as the study population. In addition, through the process of data acquisition and analysis, 2 knees were lost to follow-up, and complete pre- and postoperative data up to 2 years were not available for 6 knees. Consequently, patient data obtained from 38 knees in 38 patients were subjected to the analysis of this study,
Table 1. Demographic Characteristics and Radiographic Results

\begin{tabular}{ll}
\hline Sex $(\mathrm{F} / \mathrm{M})$ & $20 / 18$ \\
Age $(\mathrm{y})$ & $17.6 \pm 3.8(13$ to 29$)$ \\
TT-ME distance $(\mathrm{mm})$ & $14.4 \pm 4.6(4.8$ to 25.9$)$ \\
TT-TG distance $(\mathrm{mm})$ & $16.5 \pm 3.5(7.6$ to 21.8$)$ \\
Tilting angle $\left.{ }^{\circ}\right)$ & $17.2 \pm 7.2(6$ to 45$)$ \\
Lateral shift $(\%)$ & $19.9 \pm 10.1(-11.5$ to 48$)$ \\
Insall-Salvati ratio & $1.16 \pm 0.18(0.77$ to 1.55$)$ \\
Caton-Deschamps index & $1.21 \pm 0.19(1.66$ to 0.79$)$ \\
Dejour trochlear classification $(\mathrm{n})$ & A: $7, \mathrm{~B}: 7, \mathrm{C}: 15, \mathrm{D}: 9$ \\
\hline
\end{tabular}

NOTE. Data are mean \pm standard deviation (range) unless noted otherwise.

TT-ME, tibial tubercle-midepicondylar; TT-TG, tibial tubercletrochlear groove.

with a follow-up rate of $82.6 \%$ (38 of 46 knees). There were 20 females $(53 \%)$ and 18 males $(47 \%)$ with the age at surgery ranging from 13 to 29 years (mean $17.6 \pm 3.8)$. The postoperative follow-up period ranged from 24 to 60 months (mean $29.1 \pm 9.9$ ).

The demographic characteristics and radiographic assessment results are summarized in Table 1. TT-ME and TT-TG distance values in the study population ranged from 4.8 to $25.9 \mathrm{~mm}$ (mean 14.4) and 7.6 to $21.8 \mathrm{~mm}$ (mean 16.5), respectively (Table 1).

\section{Reliability Assessment for CT Measurement}

To determine the intra- and interobserver reliabilities of the CT measurements, 2 examiners (T.I. and S.K.) independently evaluated $\mathrm{CT}$ images of the initial 10 knees. Measurements were performed for TT-ME distance and TT-TG distance twice, with a time interval of 2 weeks. The intra- and interobserver reliabilities for the measurements of these radiographic distances were evaluated via intraclass correlation coefficients (ICCs). The ICC values calculated for the intraobserver reliabilities in TT-ME and TT-TG distance measurements were $0.89 / 0.91$ and $0.88 / 0.86$ (T.I./S.K.), and interobserver reliabilities were 0.96 and 0.88 , respectively. Based on this excellent reliability confirmed by the reliability analysis, measurement results by a single examiner (T.I.) were adopted and subjected to analysis in the present study.

\section{Analysis of Postoperative Recurrent Patellar Instability}

During the study period, 8 of the 38 knees $(21.1 \%)$ were deemed to have postoperative recurrent patellar instability. Among these 8 cases, recurrent dislocation occurred in 1 knee $(2.6 \%)$, and recurrent (or residual) apprehension and J-sign were identified in 3 and 4 knees $(7.9 \%$ and $10.5 \%)$, respectively.

As for the ROC curve analysis, the AUC size of TT-ME distance was 0.853 (95\% confidence interval [CI] 0.721 to $0.955, P=.002$ ), and the optimal cutoff value was $18.1 \mathrm{~mm}$. The AUC size of TT-TG distance was 0.655 


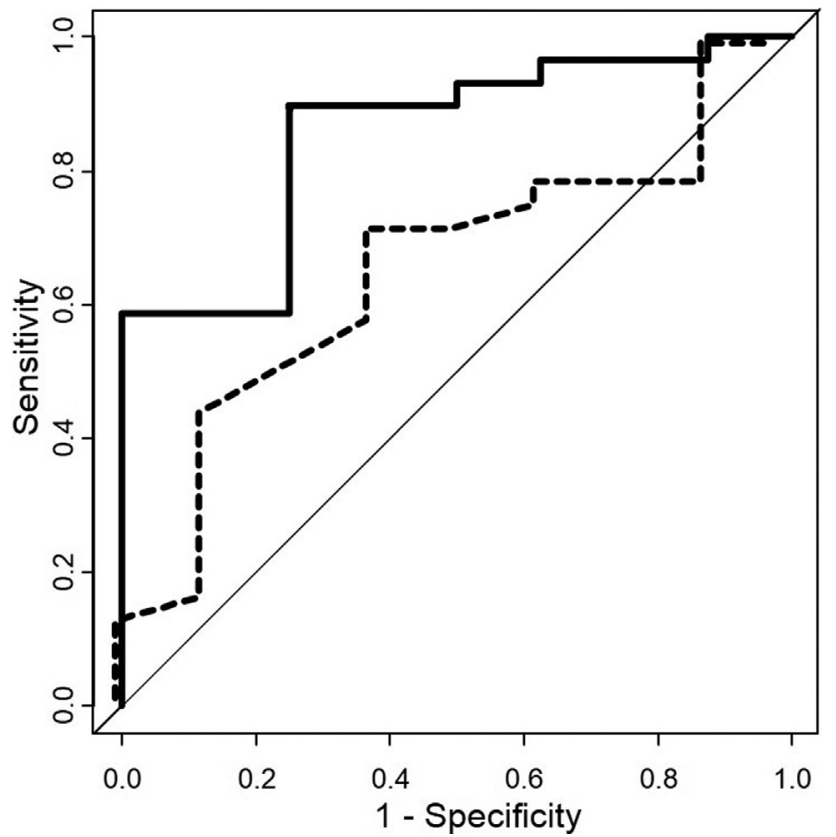

Fig 2. Comparison in receiver operator characteristics curve between tibial tubercle-midepicondyle (TT-ME) and tibial tubercle-trochlear groove (TT-TG) distances. The area under the curve size of TT-ME distance (solid line) is significantly larger than that of TT-TG distance (dotted line), indicating superiority of TT-ME distance to TT-TG distance as an indicator predicting postoperative recurrence of patellar instability. Solid line, TT-ME distance; dotted line, TT-TG distance.

(95\% CI 0.443 to $0.867, P=.184)$, and the cutoff value was $17.3 \mathrm{~mm}$. Consequently, a statistically significant difference was found between the AUC of the 2 distances $(P=.001)$, indicating superiority of TT-ME distance to TT-TG distance as an indicator predicting postoperative recurrence of patellar instability (Fig 2).

The univariable analysis for radiological factors demonstrated that the TT-ME distance was significantly associated with postoperative recurrent patellar instability (odds ratio $1.42,95 \%$ CI 1.079 to $1.868, P=.012$ ), whereas the TT-TG distance did not exhibit significant association $(P=.316)$. All other factors such as the sulcus angle did not correlate with recurrent instability (Table 2). The subsequent multivariable analysis for the TT-ME distance and patellar lateral shift $(P=.1$ in the univariable analysis) again revealed that only the TT-ME distance was shown to be a significant risk factor (odds ratio $2.412,95 \%$ CI 1.065 to $5.463, P=.035$ ) associated with postoperative recurrence of patellar instability (Table 3).

\section{Discussion}

In the present study, the TT-ME distance was proposed as an alternative to the conventionally used TT-TG distance for the assessment of tibia tubercle lateralization. In the comparison of the TT-TG and
Table 2. Results of the Univariate Logistic Regression Analysis of Risk Factors for Postoperative Patellar Instability

\begin{tabular}{|c|c|c|c|}
\hline Risk Factor & Odds Ratio & $\begin{array}{c}95 \% \text { Confidence } \\
\text { Interval } \\
\end{array}$ & $P$ Value \\
\hline TT-ME distance & 1.420 & 1.079 to 1.868 & $\overline{0.012}$ \\
\hline TT-TG distance & 1.127 & 0.892 to 1.424 & 0.316 \\
\hline Sulcus angle & 1.060 & 0.988 to 1.138 & 0.106 \\
\hline Congruence angle & 1.011 & 0.960 to 1.065 & 0.688 \\
\hline Tilting angle & 1.082 & 0.974 to 1.202 & 0.142 \\
\hline Lateral shift & 1.084 & 0.991 to 1.186 & 0.078 \\
\hline Insall-Salvati index & 2.154 & 0.027 to 169.447 & 0.731 \\
\hline $\begin{array}{l}\text { Caton-Deschamps } \\
\text { index }\end{array}$ & 0.770 & 0.21 to 47.501 & 0.901 \\
\hline Dejour classification & 0.600 & 0.930 to 3.878 & 0.592 \\
\hline
\end{tabular}

TT-ME distances as a factor influencing the surgical outcome, the TT-ME distance was shown to be a significantly more reliable indicator to predict a stabilizing effect attained by isolated MPFL reconstruction $(P=.001)$.

In the surgical management of patellar instability, MPFL reconstruction has generally been adopted as the principle surgical option. ${ }^{1-7}$ A recent level V article reported survey results derived from a group of 35 knee experts (International Patellofemoral Study Group), which indicated that MPFL reconstruction is the most favored procedure, with $77 \%$ to $86 \%$ agreement. ${ }^{19}$ Although the reported surgical results are mostly favorable, postoperative recurrent instability has been encountered at rates ranging from $5 \%$ to $18 \% .^{2,3,8,18}$ Surgical failure after isolated MPFL reconstruction is attributed to unrecognized or uncorrected bony morphological etiologies such as lateralized tibial tubercle. Consequently, combined bony procedures addressing these morphological abnormalities are advocated to improve surgical efficacy and outcome. $^{16-18}$

Among the risk factors for recurrent instability after MPFL reconstruction described above, lateralization of the tibial tubercle with resultant lateral deviation of the extensor vector is thought to be an important factor affecting the outcome of isolated medial soft tissue procedures. Conventionally, TT-TG distance on CT evaluation proposed by Dejour et al. ${ }^{11}$ has been used for quantitative assessment of lateralization of the tibial tubercle. They compared this distance between patients with patellar instability and control subjects and

Table 3. Results of the Multivariate Logistic Regression Analysis of Risk Factors for Postoperative Patellar Instability

\begin{tabular}{lccc}
\hline & \multicolumn{3}{c}{$95 \%$ Confidence } \\
\multicolumn{1}{c}{ Risk Factor } & Odds Ratio & Interval & $P$ Value \\
\hline TT-ME distance & 2.412 & 1.065 to 5.463 & 0.035 \\
Lateral shift & 1.156 & 0.897 to 1.489 & 0.262 \\
\hline
\end{tabular}

TT-ME, tibial tubercle-midepicondylar. 
identified $20 \mathrm{~mm}$ to be the threshold when measuring the TT-TG distance. ${ }^{11}$ Subsequently, various cutoff values for the requirement of concomitant distal realignment have been reported, ranging from 12 to 20 $\mathrm{mm}^{13,20-23}$; however, a consensus for the cutoff value warranting a combined distal realignment procedure has not yet been reached.

A number of studies have examined the significance of the TT-TG distance for evaluating etiologies and determining surgical options for knees with patellar instability. ${ }^{11,12,24}$ Although this distance has been shown to be related to abnormal patellar tracking and instability symptoms, some studies raised concerns regarding the reliability and clinical utility of the TT-TG distance. ${ }^{25-37}$ Various factors, such as patient size, CT examination conditions, and bony morphology, have been shown to affect the measurement reliability. ${ }^{27,28}$ As for the relationship between the degree of knee flexion during CT examination and the TT-TG distance, it has been shown that the measured TT-TG distance values are variable depending on the knee flexion angle during imaging. ${ }^{27,34}$ Difficulty in identifying the bottom of the trochlear groove ${ }^{11}$ and deviation of the position of the trochlear groove in the patient population also impair the measurement accuracy. ${ }^{35}$

To overcome the aforementioned shortcomings associated with TT-TG distance evaluation, alternative radiological distances, such as tibial tubercle-posterior cruciate ligament (TT-PCL) distance, have been proposed, and their utilities have been tested and reported in the recent literatures. ${ }^{25,29-31}$ However, TT-PCL distance represents rotational malalignment of the tibia, and the relationship between the femoral trochlea and tibial tubercle is not taken into consideration.

This study proposes the TT-ME distance as an alternative prognostic factor to the TT-TG distance after MPFL reconstruction. In assessment of the TT-ME distance, the midpoint of the transepicondylar line was adopted as a proximal reference point. This point has been conventionally used as an origin of the coordinate system of the distal femur ${ }^{38}$ and proven to be a reliable anatomic reference. ${ }^{39}$ In addition, the problem associated with a deviated trochlear groove can be overcome with this measurement method. These theoretical advantages of the TT-ME distance over the TT-TG distance were supported by the results of the present clinical study.

\section{Limitations}

The present study has some limitations. Assessment of recurrent instability was made based on 3 clinical findings (redislocation, apprehension sign, and J-sign) alone, and other clinical outcome measures, such as the Kujala score, were not used in the analysis. The cutoff value of the TT-TG distance for considering concomitant tibial tubercle transfer was determined as $22 \mathrm{~mm}$ in this study, whereas the cutoff value of $20 \mathrm{~mm}$ was adopted in some studies. ${ }^{11,12}$ The relatively high failure rate $(21 \%)$ in this study may be attributed to the strict criteria for recurrent instability as well as the surgical option in our practice during the study period. CT measurement was performed on axial images of the knee in extension; therefore, the measurement results can be different from images obtained with other flexion angles. The measurement results by a single examiner were subjected to the analysis. Although high reliability (ICC 0.88 to 0.96 ) was attained for the measurement of the distances, measurements by multiple examiners may improve the accuracy of the data acquisition. In addition, comparison of the TT-ME distance values measured in the patellar instability and normal control groups needs to be performed to validate the significance of the TT-ME in evaluation of the risk factor for patellar instability. Finally, the sample size $(\mathrm{N}=38)$ is small, and the follow-up period (minimum of 2 years) is too short. Therefore, the results of this study are not robust enough to establish an optimal guideline determining the surgical option for patellar instability.

\section{Conclusions}

Analysis of our patient population undergoing isolated MPFL reconstruction showed that the TT-ME distance was a significantly better indicator than the TT-TG distance to predict the risk for recurrent instability after isolated MPFL reconstruction performed for patellar instability.

\section{References}

1. Drez D Jr, Edwards TB, Williams CS. Results of medial patellofemoral ligament reconstruction in the treatment of patellar dislocation. Arthroscopy 2001;17:298-306.

2. Matsushita T, Kuroda R, Oka S, Matsumoto T, Takayama K, Kurosaka M. Clinical outcomes of medial patellofemoral ligament reconstruction in patients with an increased tibial tuberosity-trochlear groove distance. Knee Surg Sports Traumatol Arthrosc 2014;22:2438-2444.

3. Kita K, Tanaka Y, Toritsuka Y, et al. Factors affecting the outcomes of double-bundle medial patellofemoral ligament reconstruction for recurrent patellar dislocations evaluated by multivariate analysis. Am J Sports Med 2015;43:2988-2996.

4. Nomura E, Inoue M. Surgical technique and rationale for medial patellofemoral ligament reconstruction for recurrent patellar dislocation. Arthroscopy 2003;19:E47.

5. Wagner D, Pfalzer F, Hingelbaum S, Huth J, Mauch F, Bauer G. The influence of risk factors on clinical outcomes following anatomical medial patellofemoral ligament (MPFL) reconstruction using the gracilis tendon. Knee Surg Sports Traumatol Arthrosc 2013;21:318-324.

6. Enderlein D, Nielsen T, Christiansen SE, Fauno P, Lind M. Clinical outcome after reconstruction of the medial patellofemoral ligament in patients with recurrent patella 
instability. Knee Surg Sports Traumatol Arthrosc 2014;22: 2458-2464.

7. Nelitz M, Dreyhaupt J, Reichel H, Woelfle J, Lippacher S. Anatomic reconstruction of the medial patellofemoral ligament in children and adolescents with open growth plates: surgical technique and clinical outcome. Am J Sports Med 2013;41:58-63.

8. Shah JN, Howard JS, Flanigan DC, Brophy RH, Carey JL, Lattermann C. A systematic review of complications and failures associated with medial patellofemoral ligament reconstruction for recurrent patellar dislocation. Am J Sports Med 2012;40:1916-1923.

9. Valkering KP, Rajeev A, Caplan N, Tuinebreijer WE, Kader DF. An evaluation of the effectiveness of medial patellofemoral ligament reconstruction using an anatomical tunnel site. Knee Surg Sports Traumatol Arthrosc 2017;25:3206-3212.

10. Colvin AC, West RV. Patellar instability. J Bone Joint Surg Am 2008;90:2751-2762.

11. Dejour H, Walch G, Nove-Josserand L, Guier C. Factors of patellar instability: an anatomic radiographic study. Knee Surg Sports Traumatol Arthrosc 1994;2:19-26.

12. Williams AA, Elias JJ, Tanaka MJ, et al. The relationship between tibial tuberosity-trochlear groove distance and abnormal patellar tracking in patients with unilateral patellar instability. Arthroscopy 2016;32:55-61.

13. Schottle PB, Fucentese SF, Romero J. Clinical and radiological outcome of medial patellofemoral ligament reconstruction with a semitendinosus autograft for patella instability. Knee Surg Sports Traumatol Arthrosc 2005;13: 516-521.

14. Weber AE, Nathani A, Dines JS, et al. An algorithmic approach to the management of recurrent lateral patellar dislocation. J Bone Joint Surg Am 2016;98:417-427.

15. Stephen JM, Dodds AL, Lumpaopong P, Kader D, Williams A, Amis AA. The ability of medial patellofemoral ligament reconstruction to correct patellar kinematics and contact mechanics in the presence of a lateralized tibial tubercle. Am J Sports Med 2015;43: 2198-2207.

16. Burnham JM, Howard JS, Hayes CB, Lattermann C. Medial patellofemoral ligament reconstruction with concomitant tibial tubercle transfer: A systematic review of outcomes and complications. Arthroscopy 2016;32: 1185-1195.

17. Franciozi CE, Ambra LF, Albertoni LJB, et al. Anteromedial tibial tubercle osteotomy improves results of medial patellofemoral ligament reconstruction for recurrent patellar instability in patients with tibial tuberositytrochlear groove distance of 17 to $20 \mathrm{~mm}$. Arthroscopy 2019;35:566-574.

18. Longo UG, Berton A, Salvatore G, et al. Medial patellofemoral ligament reconstruction combined with bony procedures for patellar instability: Current indications, outcomes, and complications. Arthroscopy 2016;32: $1421-1427$.

19. Liu JN, Steinhaus ME, Kalbian IL, et al. Patellar instability management: A survey of the International Patellofemoral Study Group. Am J Sports Med 2018;46:3299-3306.

20. Koeter S, Diks MJ, Anderson PG, Wymenga AB. A modified tibial tubercle osteotomy for patellar maltracking: results at two years. J Bone Joint Surg $\mathrm{Br}$ 2007;89:180-185.

21. Mellecker S, Ebinger T, Butler P, Albright J. SouthwickFulkerson osteotomy with intraoperative femoral nerve guidance. Iowa Orthop J 2013;33:90-96.

22. Tecklenburg K, Feller JA, Whitehead TS, Webster KE, Elzarka A. Outcome of surgery for recurrent patellar dislocation based on the distance of the tibial tuberosity to the trochlear groove. J Bone Joint Surg Br 2010;92: 1376-1380.

23. Muneta T, Sekiya I, Tsuchiya M, Shinomiya K. A technique for reconstruction of the medial patellofemoral ligament. Clin Orthop Relat Res 1999;359:151-155.

24. Caplan N, Lees D, Newby M, et al. Is tibial tuberositytrochlear groove distance an appropriate measure for the identification of knees with patellar instability? Knee Surg Sports Traumatol Arthrosc 2014;22:2377-2381.

25. Heidenreich MJ, Camp CL, Dahm DL, Stuart MJ, Levy BA, Krych AJ. The contribution of the tibial tubercle to patellar instability: Analysis of tibial tubercle-trochlear groove (TT-TG) and tibial tubercle-posterior cruciate ligament (TT-PCL) distances. Knee Surg Sports Traumatol Arthrosc 2017;25:2347-2351.

26. Hevesi M, Heidenreich MJ, Camp CL, Hewett TE, Stuart MJ, Dahm DL, Krych AJ. The recurrent instability of the patella score: A statistically based model for prediction of long-term recurrence risk after first-time dislocation. Arthroscopy 2019;35:537-543.

27. Tanaka MJ, Elias JJ, Williams AA, Carrino JA, Cosgarea AJ. Correlation between changes in tibial tuberosity-trochlear groove distance and patellar position during active knee extension on dynamic kinematic computed tomographic imaging. Arthroscopy 2015;31: 1748-1755.

28. Pennock AT, Alam M, Bastrom T. Variation in tibial tubercle-trochlear groove measurement as a function of age, sex, size, and patellar instability. Am J Sports Med 2014;42:389-393.

29. Seitlinger G, Scheurecker G, Hogler R, Labey L, Innocenti B, Hofmann S. Tibial tubercle-posterior cruciate ligament distance: A new measurement to define the position of the tibial tubercle in patients with patellar dislocation. Am J Sports Med 2012;40:1119-1125.

30. Brady JM, Sullivan JP, Nguyen J, Mintz D, Green DW, Strickland S, Shubin Stein BE. The tibial tubercle-totrochlear groove distance is reliable in the setting of trochlear dysplasia, and superior to the tibial tubercle-toposterior cruciate ligament distance when evaluating coronal malalignment in patellofemoral instability. Arthroscopy 2017;33:2026-2034.

31. Anley CM, Morris GV, Saithna A, James SL, Snow M. Defining the role of the tibial tubercle-trochlear groove and tibial tubercle-posterior cruciate ligament distances in the work-up of patients with patellofemoral disorders. Am J Sports Med 2015:43:1348-1353.

32. Alemparte J, Ekdahl M, Burnier L, et al. Patellofemoral evaluation with radiographs and computed tomography scans in 60 knees of asymptomatic subjects. Arthroscopy 2007;23:170- 177 .

33. Jones RB, Barlett EC, Vainright JR, Carroll RG. CT determination of tibial tubercle lateralization in patients 
presenting with anterior knee pain. Skelet Radiol 1995;24: 505-509.

34. Diederichs G, Kohlitz T, Kornaropoulos E, Heller MO, Vollnberg B, Scheffler S. Magnetic resonance imaging analysis of rotational alignment in patients with patellar dislocations. Am J Sports Med 2013;41:51-57.

35. Tscholl PM, Antoniadis A, Dietrich TJ, Koch PP, Fucentese SF. The tibial-tubercle trochlear groove distance in patients with trochlear dysplasia: The influence of the proximally flat trochlea. Knee Surg Sports Traumatol Arthrosc 2016;24:2741-2747.

36. Boutris N, Delgado DA, Labis JS, McCulloch PC, Lintner DM, Harris JD. Current evidence advocates use of a new pathologic tibial tubercle-posterior cruciate ligament distance threshold in patients with patellar instability. Knee Surg Sports Traumatol Arthrosc 2018;26: 2733-2742.

37. Hinckel BB, Gobbi RG, Kihara Filho EN, Demange MK, Pecora JR, Camanho GL. Patellar tendon-trochlear groove angle measurement: A new method for patellofemoral rotational analyses. Orthop J Sports Med 2015;3: 2325967115601031.

38. Grood ES, Suntay WJ. A joint coordinate system for the clinical description of three-dimensional motions: application to the knee. J Biomech Eng 1983;105:136-144.

39. Tsujimoto K, Kurosaka M, Yoshiya S, Mizuno K. Radiographic and computed tomographic analysis of the position of the tibial tubercle in recurrent dislocation and subluxation of the patella. Am J Knee Surg 2000;13: 83-88. 